\title{
Calibration of the Soft X-ray Telescopes (SXT) Onboard the ASTRO-H Satellite
}

\author{
Yang Soong ${ }^{\text {a }}$, Takashi Okajima, and Peter J. Serlemitsos \\ NASA Goddard Space Flight Center, 20771 \\ ${ }^{\mathrm{a} C R E S S T / U S R A}$
}

\begin{abstract}
ASTRO-H is an astrophysics satellite dedicated for non-dispersive X-ray spectroscopic study on selective celestial X-ray sources. Among the onboard instruments there are four Wolter-I X-ray mirrors of their reflectors' figure in conical approximation. Two of the four are soft X-ray mirrors ${ }^{1}$, of which the energy range is from a few hundred $\mathrm{eV}$ to $15 \mathrm{keV}$. The focal point instruments will be a calorimeter (SXS) and a CCD camera (SXI), respectively. The mirrors were in quadrant configuration with photons being reflected consecutively in the primary and secondary stage before landing on the focal plane of $5.6 \mathrm{~m}$ away from the interface between the two stages. The reflectors of the mirror are made of heat-formed aluminum substrate of the thickness gauged of $152 \mu \mathrm{m}, 229 \mu \mathrm{m}$, and $305 \mu \mathrm{m}$ of the alloy $5052 \mathrm{H}-19$, followed by epoxy replication on gold-sputtered smooth Pyrex cylindrical mandrels to acquire the X-ray reflective surface. The epoxy layer is $10 \mu \mathrm{m}$ nominal and surface gold layer of $0.2 \mu \mathrm{m}$. Improvements on angular response over its predecessors, e.g. Astro-E1/Suzaku mirrors, come from error reduction on the figure, the roundness, and the grazing angle/radius mismatching of the reflecting surface, and tighter specs and mechanical strength on supporting structure to reduce the reflector positioning and the assembly errors.

Each soft x-ray telescope (SXT), FM1 or FM2, were integrated from four independent quadrants of mirrors. The stray-light baffles, in quadrant configuration, were mounted onto the integrated mirror. Thermal control units were attached to the perimeter of the integrated mirror to keep the mirror within operating temperature in space. The completed instrument went through a series of optical alignment, thus made the quadrant images confocal and their optical axes in parallel to achieve highest throughput possible.

Environmental tests were carried out, and optical quality of the telescopes has been confirmed. The optical and x-ray calibrations also include: angular resolution, effective area in the energy range of $\sim 0.4-12 \mathrm{keV}$, off-axis response, etc. Some of those are being carried out by our counterpart at JAXA/ISAS, Japan. We report the calibration results of the FM1 and FM2 that were obtained at Goddard Space Flight Center.
\end{abstract}

Keywords: X-ray Optics, X-ray Telescope, X-ray Astrophysical Instrument

\section{INTRODUCTION}

Conical approximation of the Wolter-I type X-ray imager has been a major tool for spectroscopic study in the last quarter of century since the launch of a sounding rocket experiment to study SN1987A. The high throughput, essential for the spectroscopic study, of the telescope is provided by using thin-foil reflectors, and the continuous improvement of angular response on X-ray image is accomplished by switching the substrate surface smoothing scheme from earlier lacquer-dipping to later epoxy replication that reduced the mid-frequency waviness ${ }^{2}$. The low micro-roughness of the surface produces specular reflection of X-rays, using high $\mathrm{Z}$ material such as Au, Pt, or Ir as the reflecting surface. The energy range coverage can be up to $15 \mathrm{keV}$ with shallower grazing incidence. Despite the continuous improvement over the last two decades only from 4 to 1.7 arc-minutes, the demand of better angular resolution of such thin-foil mirrors is high. The current image quality is still far from achieving the conical approximation limit, which is about 0.2 arc-minute. The difficulty comes from the relatively low mechanical integrity of the reflectors and the imprecision in the assembly/alignment scheme. The improvement of HPD from the Suzaku mirror of 2 arc-minutes to the recent test of engineering model of ASTRO-H of 1 arc-minute will be the main subject of this paper. 
Table 1 Design parameters of ASTRO-H and Suzaku

\begin{tabular}{|l|l|l|}
\hline & ASTRO-H & Suzaku \\
\hline Effective aperture diameter $(\mathrm{cm})$ & $11.6-45.0$ & $11.6-40.0$ \\
\hline Focal length $(\mathrm{m})$ & 5.6 & $4.75 / 4.50$ \\
\hline Reflector height $(\mathrm{cm})$ & 10.16 & 10.16 \\
\hline \# of reflectors & 203 & $175 / 168$ \\
\hline Mass $(\mathrm{kg})$ & 44 & 20 \\
\hline \# of mirrors $(0.4-12 \mathrm{keV})$ & 2 & $4 / 1$ \\
\hline Substrate thickness $(\mu \mathrm{m})$ & $152,229,305$ & 152 \\
\hline Epoxy thickness $(\mu \mathrm{m})$ & 10 & 25 \\
\hline Au thickness $(\AA)$ & 2000 & 2000 \\
\hline Grazing angle $\left({ }^{\circ}\right)$ & $0.15-0.59$ & $0.18-0.62 / 0.19-0.65$ \\
\hline FOV $($ arc-minute $)$ & 17 & 19 \\
\hline Expected effective area $\left(\mathrm{cm}^{2}\right)$ & $580 / 445 / 369 @$ & $450 / 250 @ 1.5 / 8.0 \mathrm{keV}(4.75 \mathrm{~m}$ \\
& $1.5 / 4.5 / 8.0 \mathrm{keV} \dagger$ & measured $)$ \\
\hline
\end{tabular}

† Beam Line Weekly Report No. 250, by Y. Maeda, et al., July 8 - 14, 2013

\section{THE SCHEME}

Error terms of image at the quadrant level can be categorized with quality of reflectors and assembly. For those coming from reflectors are axial figure, roundness, radius/grazing angle matching, surface roughness, and microroughness of the reflecting surface. Assembly errors come from precision of the holding structure, alignment, thermal, and under the gravity influence/image stability. Error reduction from Suzaku to ASTRO-H is listed in Table 2.

Table 2 Error terms at quadrant level. The dominant term of error is the reflector positioning (alignment)

\begin{tabular}{|l|l|l|}
\hline & ASTRO-H & Suzaku \\
\hline Radius/grazing angle mismatching & $1.2-2.5 \%$ & $2.3-9.2 \%$ \\
\hline Axial figure $($ arc-minute) & 0.7 & 1 \\
\hline Mid-frequency, $\mu \mathrm{m}-\mathrm{mm}(\AA)$ & $4-6$ & $4-6$ \\
\hline Micro-roughness $(\AA)$ & 5 & 5 \\
\hline & & \\
\hline Alignment groove position drift $(\mu \mathrm{m})$ & $<5$ & $<40$ \\
\hline Alignment groove random $(\mu \mathrm{m})$ & 5 & 12 \\
\hline Alignment $(\mu \mathrm{m})$ & 5 & 10 \\
\hline Focal length uncertainty $(\mathrm{cm})$ & 0.5 & $<5$ \\
\hline & & \\
\hline Image quality HPD $($ arc-minute $)$ & $1.1-1.2$ & $1.7-2.2$ \\
\hline
\end{tabular}

\subsection{Quality of Reflectors}

\subsubsection{Substrate Forming}

Forming mandrels were conically shaped with sequentially increasing radius so that to provide the complete set of substrate at all design radii. There are 71 pairs of aluminum mandrels for ASTRO-H vs. 20 pairs of quartz mandrels for Suzaku. CTE matching with the substrate during forming at $200^{\circ} \mathrm{C}$ are much improved $\left(23 \times 10^{-6} /{ }^{\circ} \mathrm{C}\right.$ vs. $\left.0.59 \times 10^{-6} /{ }^{\circ} \mathrm{C}\right)$. 
Radius/grazing angle matching: related to substrate forming scheme. For all substrate thickness, we stack 11 pieces in one forming batch. In theory only the innermost foil in each batch of forming will have the right radius/grazing angle match, the rest of the stack will have the matching error, from inner to outer foils in a particular batch, which was reduced from 9.2\%/2.3\% for Suzaku to $2.5 \% / 1.2 \%$ for ASTRO-H.

Axial figure: generally is improved from $4 \mu \mathrm{m}$ to $1-2 \mu \mathrm{m}$, due to the improvement on surface figure error of the substrate forming mandrels. RMS error of the figure slope is about 0.4 arc-minute ${ }^{3}$.

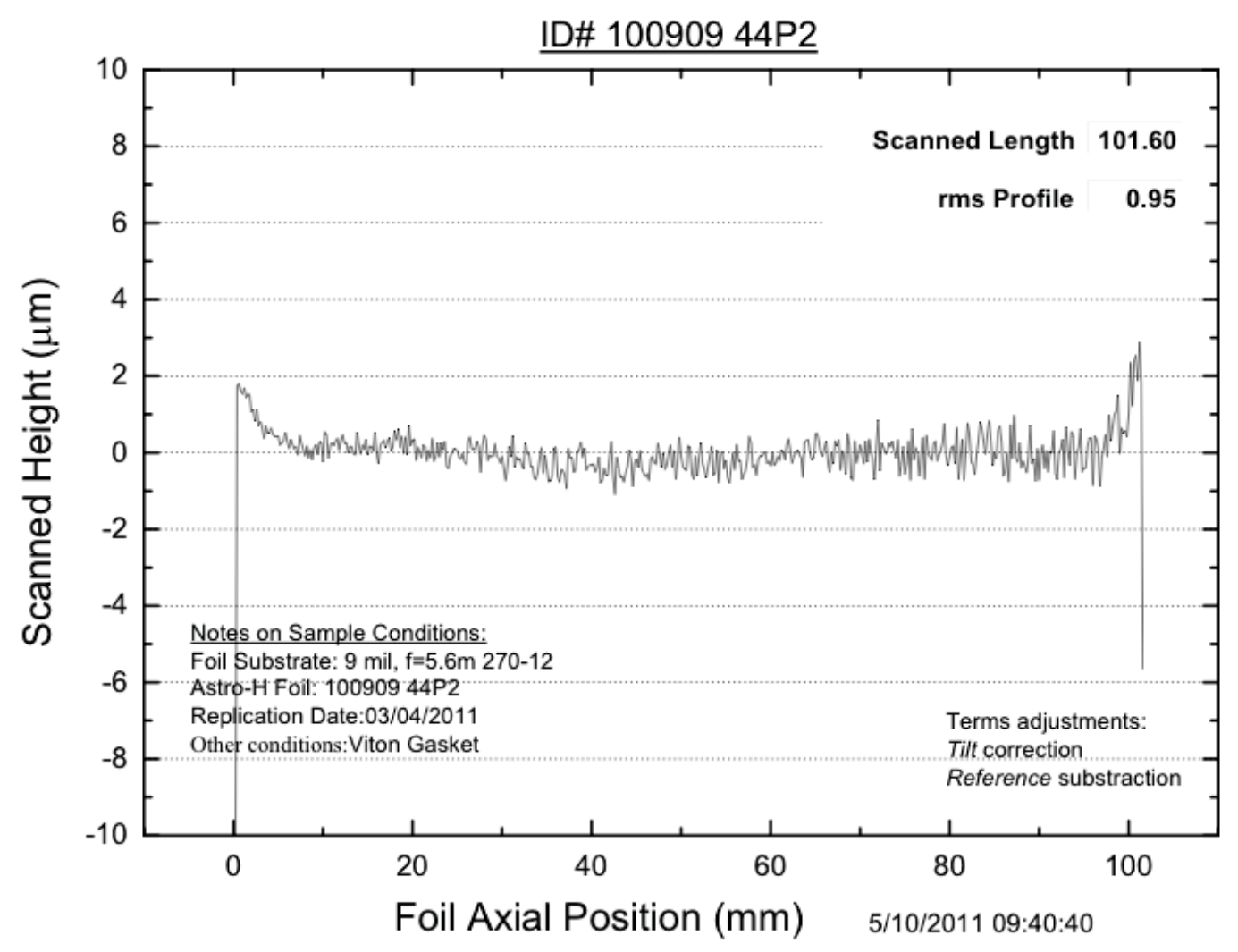

Fig.1 Axial figure of a typical reflector. Scan step is $200 \mu \mathrm{m}$

Roundness: error of $1 / 3$ arc-minute (substrate size difference $\sim+/-1$ ), which translates to $10 \mu \mathrm{m}$ in reflector edge positioning error in the alignment bar grooves. RMS of this term is on an order of 1 arc-minute, which largely a limiting factor for reaching a sub-arc-minute image quality.

Mid-frequency roughness: 4-5 $\AA$ at spatial wavelength $10^{-3}-1 \mathrm{~mm}$. Result of Zygo surface profilometer is provided in Fig. 2.

Micro-roughness: no obvious problem was implied by X-ray scattering studies.

\subsubsection{Epoxy Replication}

Although the process has been repeated for an order of 20000 times for a range of radius, a variety of Pyrex mandrel figure quality and a number of projects, The one-to-one correspondence that related the quality of finished reflector and that of the mandrel is still not totally established. Statistically speaking, the better the mandrel figure the better reflector figure will be, and the thinner the epoxy layer in the replication the better the reflector figure will be. A more rigorous 
replication mandrel screening/selecting process and thinning of the epoxy layer, from $25 \mu \mathrm{m}$ to $10 \mu \mathrm{m}$, certainly made the reflector quality superior to of those used in the Suzaku mirrors.

Improvement of reflector quality is critical to enhancing of angular resolution in the future. The answer is lying on the replication process, i.e. control the mandrel figure and precision on applying the epoxy layer, especially on the demand of larger and larger aperture mirror for spectroscopic missions. We firmly believe that this key issue can be closely approached in the next few years.

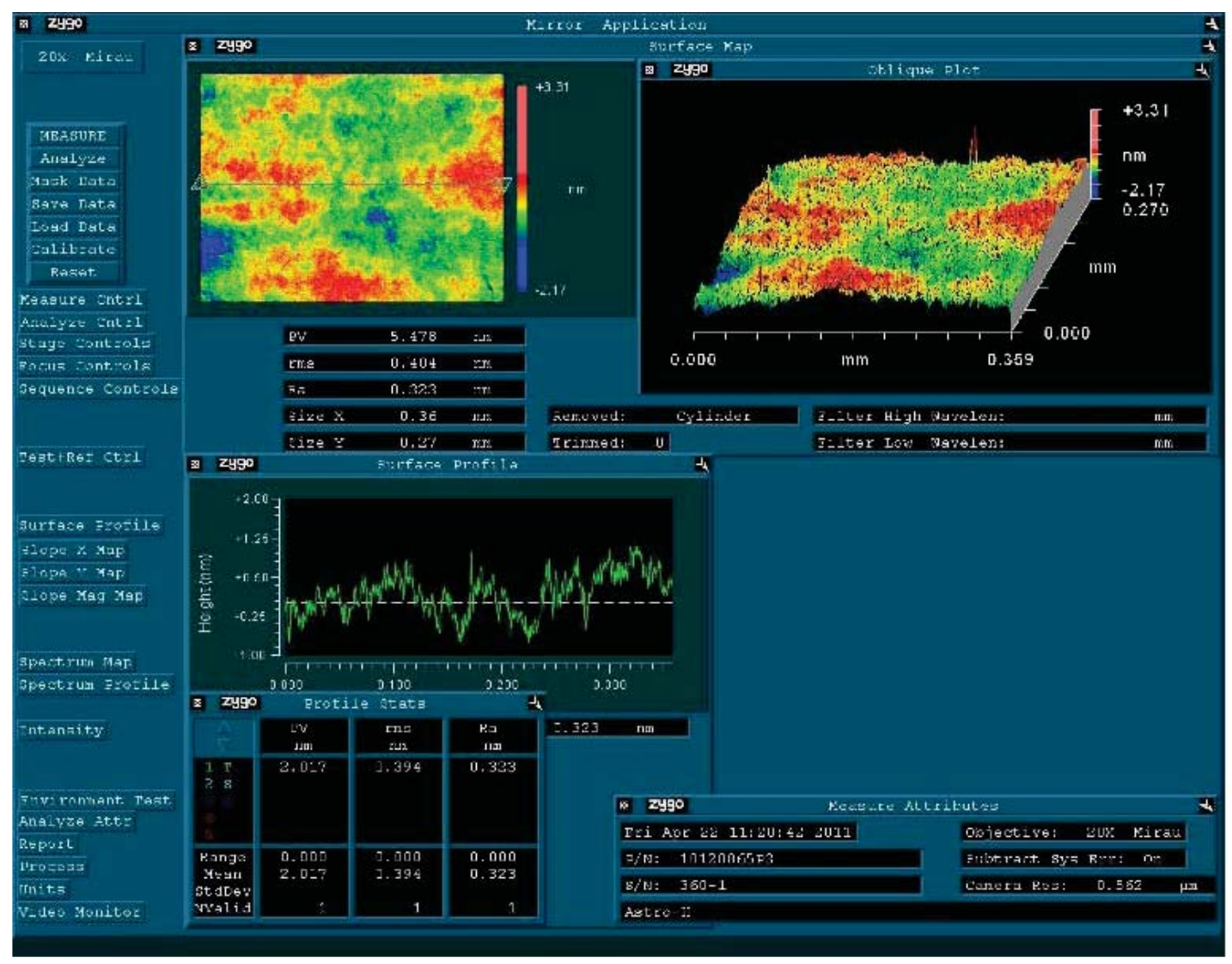

Fig. 2 Surface profile of a typical reflector

\subsection{Assembly at quadrant level}




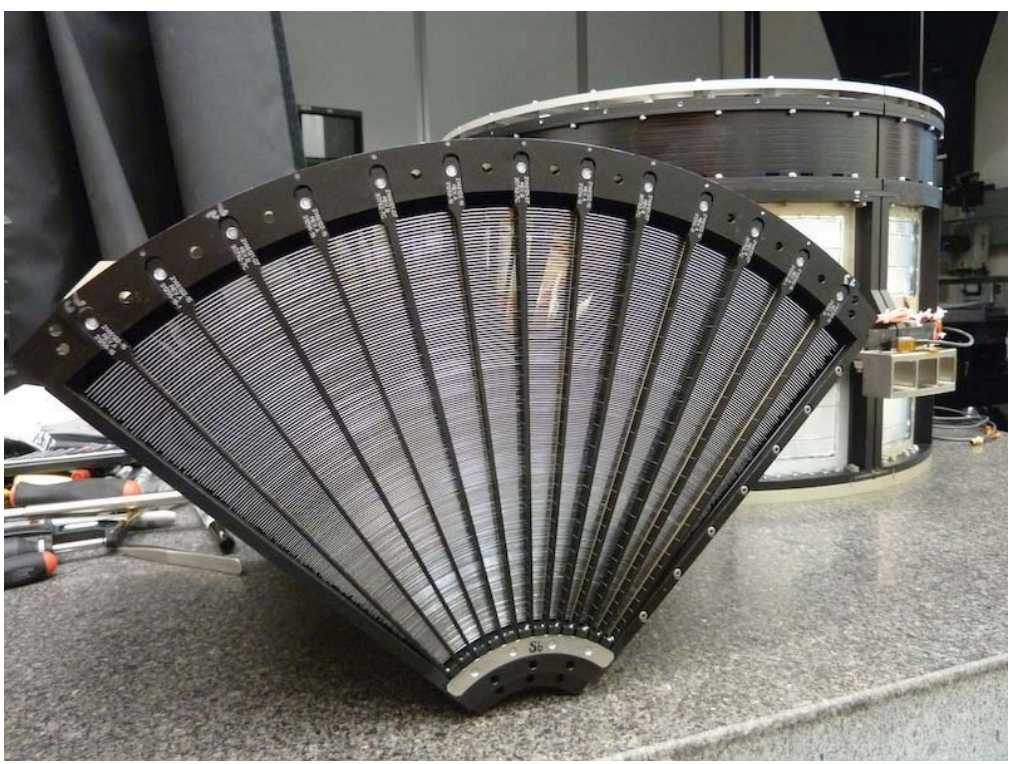

Fig. 3 ASTRO-H FM2 fully populated housing of 203 shells and the completed FM1 is in the background. There are 13 alignment/support bars, on each phase, each with 203 grooves that hold the 203 shells by the edges

\section{Holding structure:}

- alignment bars, $<5 \mu \mathrm{m}$ systematic walk of groove position from end to end; random error of the groove position: $+/-5 \mu \mathrm{m}$.

- mechanically strengthen the housing structure to resist distortion in the assembly; distortion was minimized with 3-point mount scheme at two-plane interface or by filling the gaps with Stycast 2850FT before tightening the fasteners. The rest of the connecting screw positions will be dabbed with Stycast as liquid shims in the interface. These set of screws will be tightened after the epoxy is fully cured.

8 pieces of alignment bars, with tight specs on the grooves' radial positioning and 5 support bars, with wider groove for Arathane 5753 application later for stabilizing the image, a total of 13 bars, were placed on each phase of a housing as it was prepared. 203 pieces of reflectors were prepared, i.e. selecting, sizing and cleaning, before loading into the housing. Loading was by insertion azimuthally through a side opening with a panel temporarily being removed.

Alignment was done with the setup in the Fig. 4 below. It consists of a rotation table that carries the quadrant housings and a linear translation stage that carries a 500x magnification microscope, which monitor the radial position of the alignment/support bars. The rotation stage and the linear stage carry specs at axis wobble of $\mu$ radian and linear error of $1 \mu \mathrm{m}$, respectively. After center of rotation of the turntable was set within $+/-1 \mu \mathrm{m}$, each alignment bar was adjusted to its designated radial position. Each pair of housings, primary and its matching secondary one, were tuned in this way for a complete mirror of 4 quadrants. All bars were staked with Stycast 2850FT to the inner flange of the housing, which is considered the most stable radial reference of the whole housing structure.

Pair of housings was coupled to check the image quality, throughput and focal length, optically with the horizontal beam in Fig. 5, and X-ray tests soon follow. After confirming the quality of the image, etc., all reflectors were staked with Arathane 5753 to 5 support bars and 2 end alignment bars for image stability for ground testing and space deployment. The 6 alignment bars were removed after curing of the Arathane. The remaining bars then were further staked firmly with the housing at inner and outer flanges to ensure the structural integrity of the alignment in the future assembly processes. Focal length determination was set to be within $+/-5 \mathrm{~mm}$. Minor adjustment of the angle extending between primary and secondary housings at interface was necessary, which was achieved by varying shim thickness on three points, one at inner flange and two at the outer flange, in between the two housings to the ultimate controllable thickness of $2 \mu \mathrm{m}$. 




Fig. 4 Alignment setup in polar coordinates. Precision and repeatability of the translation stage was at $1 \mu \mathrm{m}$, and wobble of the rotation axis was at $1 \mu \mathrm{rad}$
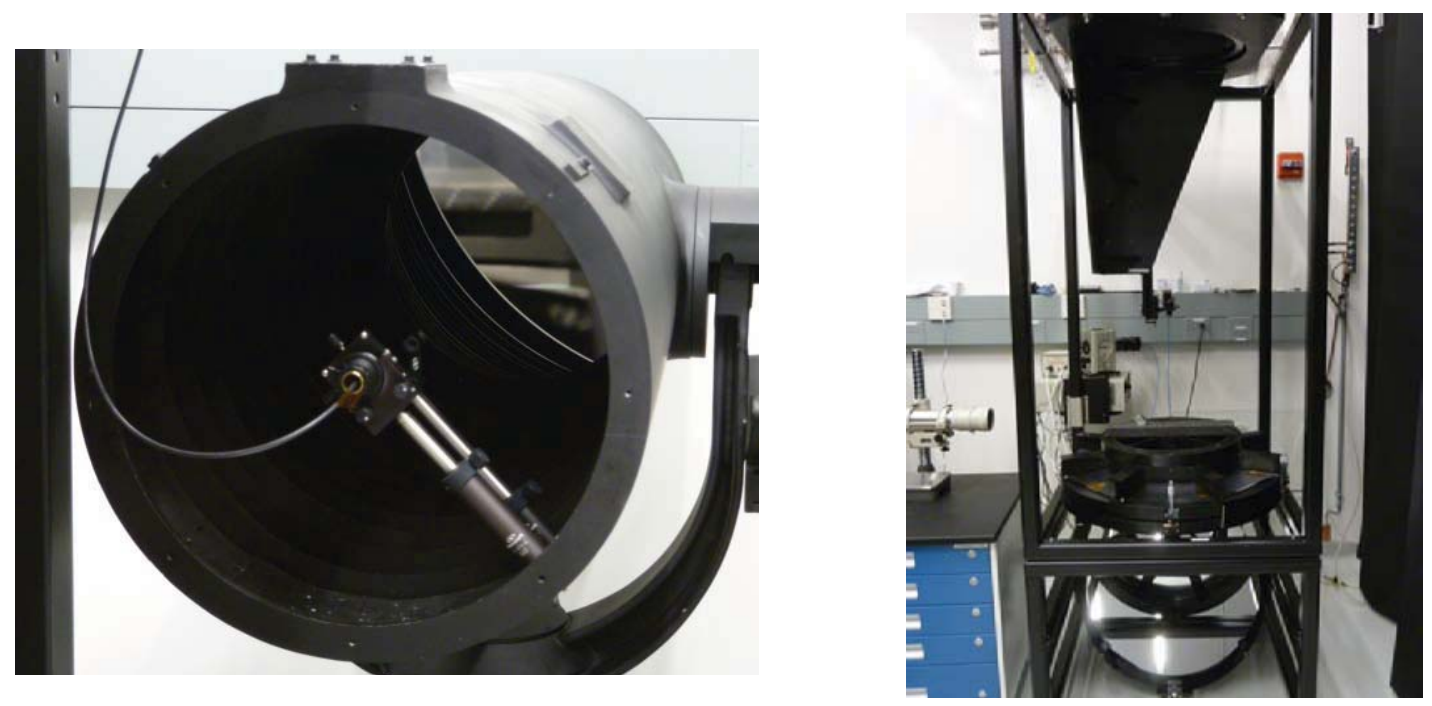

Fig. 5 The horizontal and the vertical optical beams were used for image calibration. Of the former an on-axis and the latter an off-axis parabola prime mirror. Each was calibrated to beam parallelism at 2 arc-second

Thermal: mirror quadrant was tested for thermal dependence of HPD. At $22^{\circ} \mathrm{C}+/-10^{\circ} \mathrm{C}$ the HPD changed within 1.1 +/- 0.05 arc-minute.

Vibration: FM1 was vibrated under a qualification level, and it had no significant change of the image and alignment. FM2 will go through that at the acceptance level. We expect no change afterwards as well.

\subsection{Quadrants integration into a complete mirror}

After four quadrants were calibrated satisfactorily, integration into a complete flight mirror assumes. The vertical optical beam, see in Fig. 5, was used for the purpose. Features so designed to ensure the confocality of the four quadrants and optical axes alignment are kept in the assembly and environmental tests. 
- Rings at the inner/outer radii at exiting side, so called the secondary bottom phase, of the mirror to interface with a 3-point contact on a granite table

- Check optical axes of the four quadrants with the vertical beam. Adjust the shims at 3-point contact to align the optical axes in parallel. Quadrants were moved laterally to make the four foci confocal. Mark the relative position of the neighboring quadrants with fine readable lines for repeatability. Apply the interface with Stycast 2850FT surrounding all connecting screws, and adjust quadrant position to match the existing mark lines before the Stycast congealed

- A ring on inner radial flange at the entrance side, so called the primary top phase, of the mirror was attached with Stycast 2850FT to ensure the relative positions of the quadrants were firmly kept

- Metal tabs on 8 locations at outer flange that connect the neighboring quadrants were staked with Stycast $2850 \mathrm{FT}$ and then fastened to keep the relative positions between the quadrants unvaried

- Stycast 2850FT was used in the liquid pins in between the quadrants' outer flanges in 8 places to further strengthen the bond in between the neighboring quadrants

- All elements were held with liquid shims, to achieve minimal stress in the assembled system

- Match-drilled, in four groups of interface foot (I/F as seen in the above picture at outer flange of the housings), of 2 points a group, of $90^{\circ}$ apart at the outer rim at interface of the primary and secondary housings, to be anchoring onto the top plate of the spacecraft

- Thermal control units, sensors and control circuits were attached to the mirror perimeter

- Mounting pre-collimator (PC) onto top of the primary stage, see in Fig. 6

- Later in Japan, thermal shields (TS), 0.2-micron polyimide supported by stainless wire mesh $(94 \%$ transmission) will be placed on top of PC

- Calibration with 100-m divergent X-ray beam before vibration qualification, and confirming of mechanical integrity and image quality with the same beam afterwards

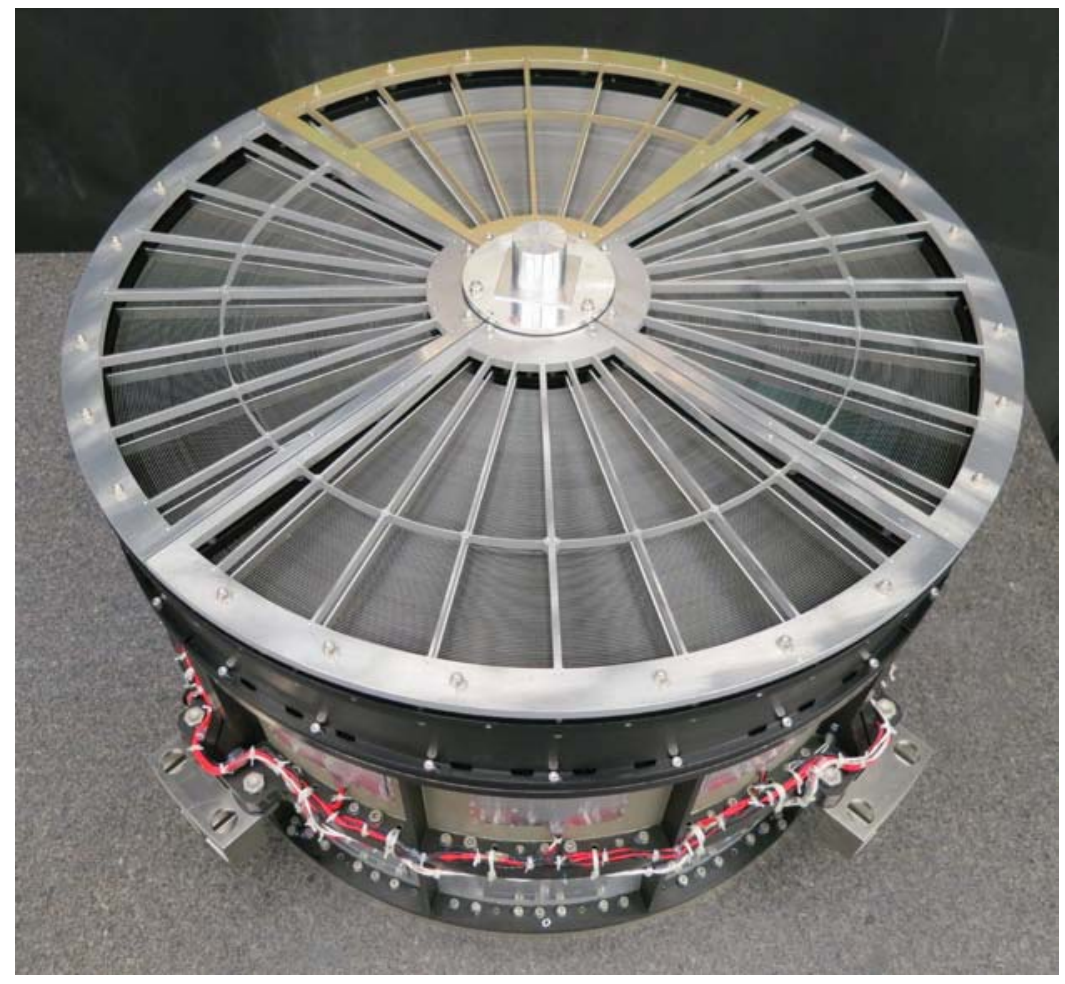

Fig. 6 ASTRO-H FM1 integrated mirror with corresponding pre-collimator (PC, from Nagoya University) on top of each quadrant, and one thermal shield frame(TS, from Nagoya University) on one of the quadrant 


\section{RESULTS}

\subsection{Optical Axes Alignment and Confocality Results}

Integrating four quadrants into a complete mirror is an important step to ensure that the quality of the individual quadrant image will be kept at its optimum. The procedures have to set up in order to guard against gravity distortion and mechanical stresses. The goal is to align the individual optical axis to be within 1'x1' box, and their foci to be located within $100 \mu \mathrm{m} \times 100 \mu \mathrm{m}$ box on the focal plane of the mirror. The error bars in these two measurements were $\pm 0.2^{\prime}$ and $\pm 20 \mu \mathrm{m}$. The results of the FM1 and FM2 are presented in the Fig. 7.
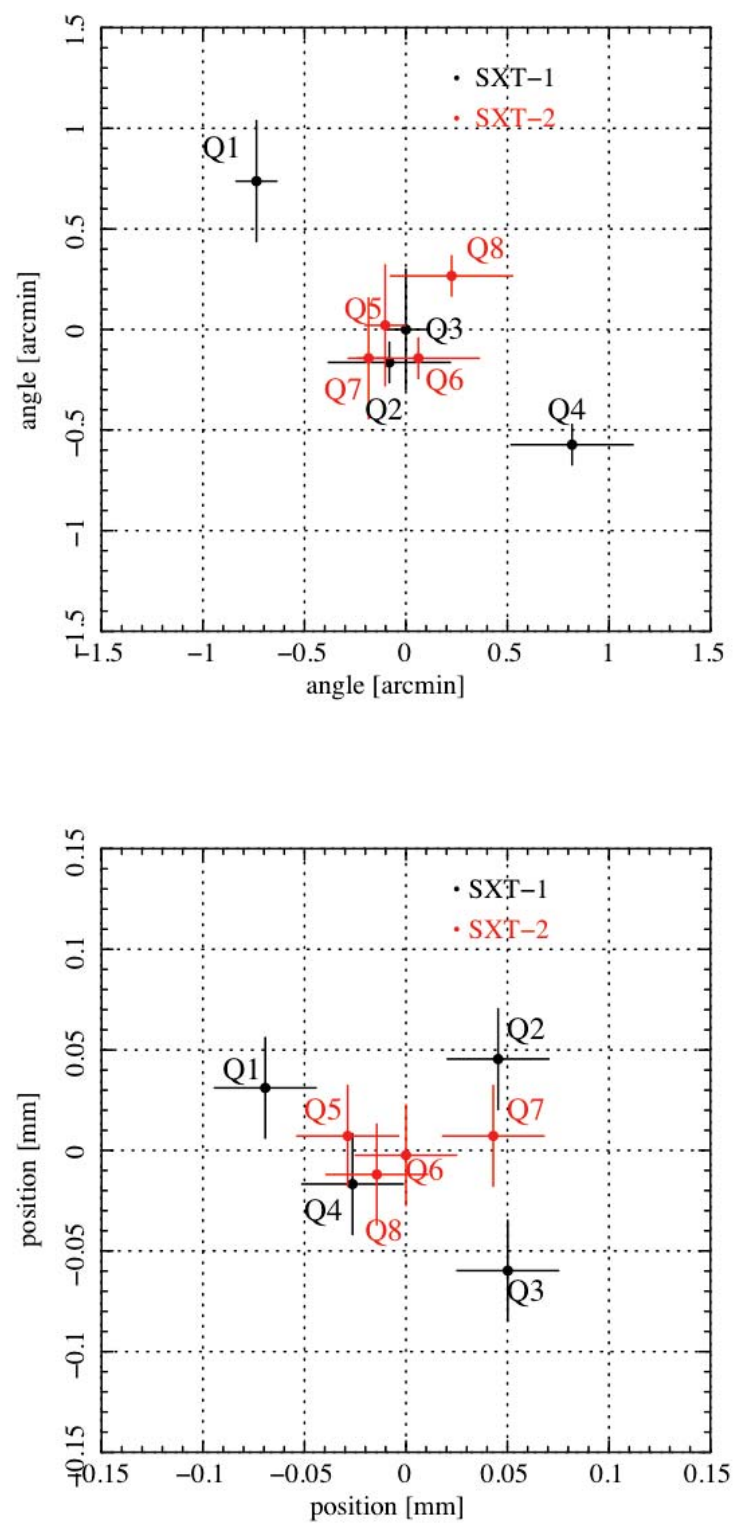

Fig. 7 Results of quadrants' optical alignment and their image confocality

\subsection{X-ray Test Results}


X-ray image of a typical flight mirror quadrant
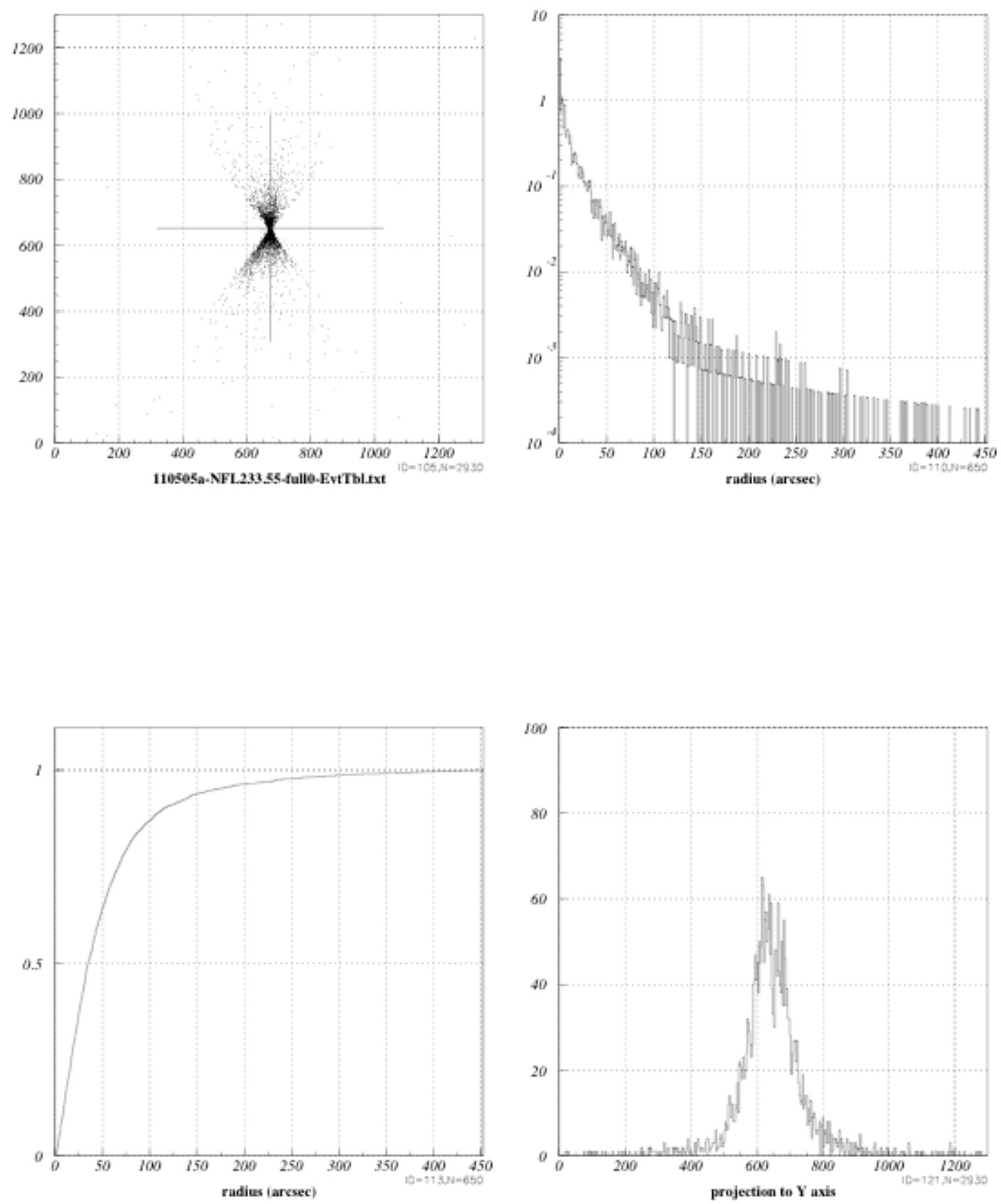
Fig. 8 X-ray test result of a typical ASTRO-H FM quadrant, clockwise from top left: X-ray image on CCD, Point Spread Function (PSF) of the image, and profile of the image projected to the abscissa, and Encircled Energy Function (EEF). HPD of the FM image is $\sim 1$ arc-minute

Sector images, a total of 12 full sectors and 2 half sectors, and the sector centroid distribution are shown in Fig. 9. Sector image is $\sim 50-60$ arc-seconds. The centroid of the sector image spread $\sim 1 \mathrm{~mm}$ on the focal plane, and the RMS of the error is $\sim 0.25$ arc-minute.
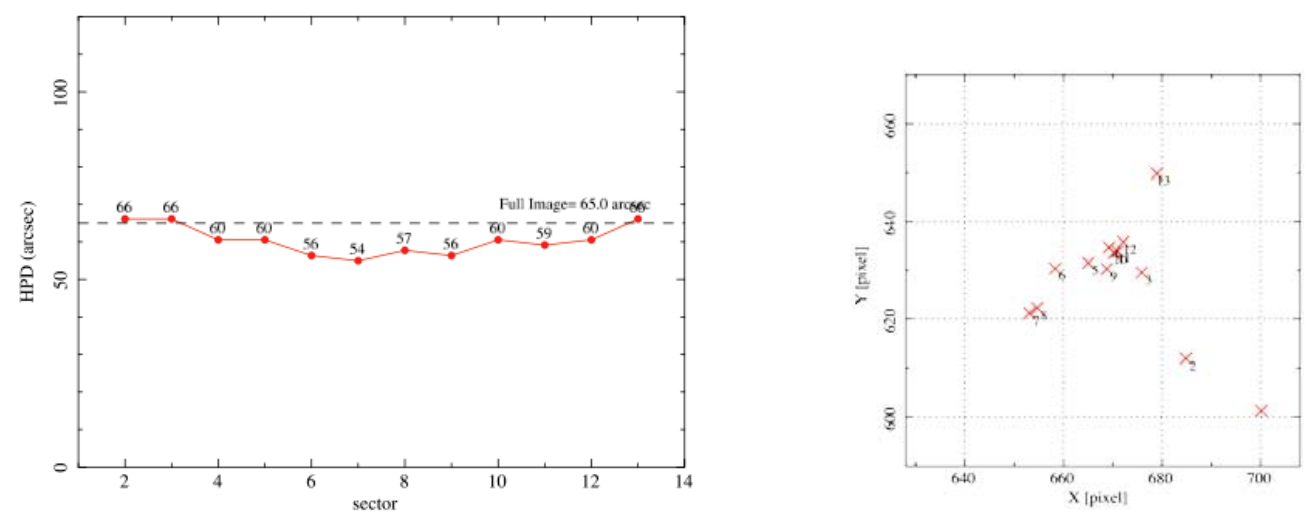

Fig. 9 The left panel, sector image HPD. 13 alignment bars divided the quadrant aperture into 12 full sectors in between and 2 halfsectors at quadrant ends; the right panel, sector centroid distribution on the focal plane, pixel size $20 \mu \mathrm{m}$ x $20 \mu \mathrm{m}$

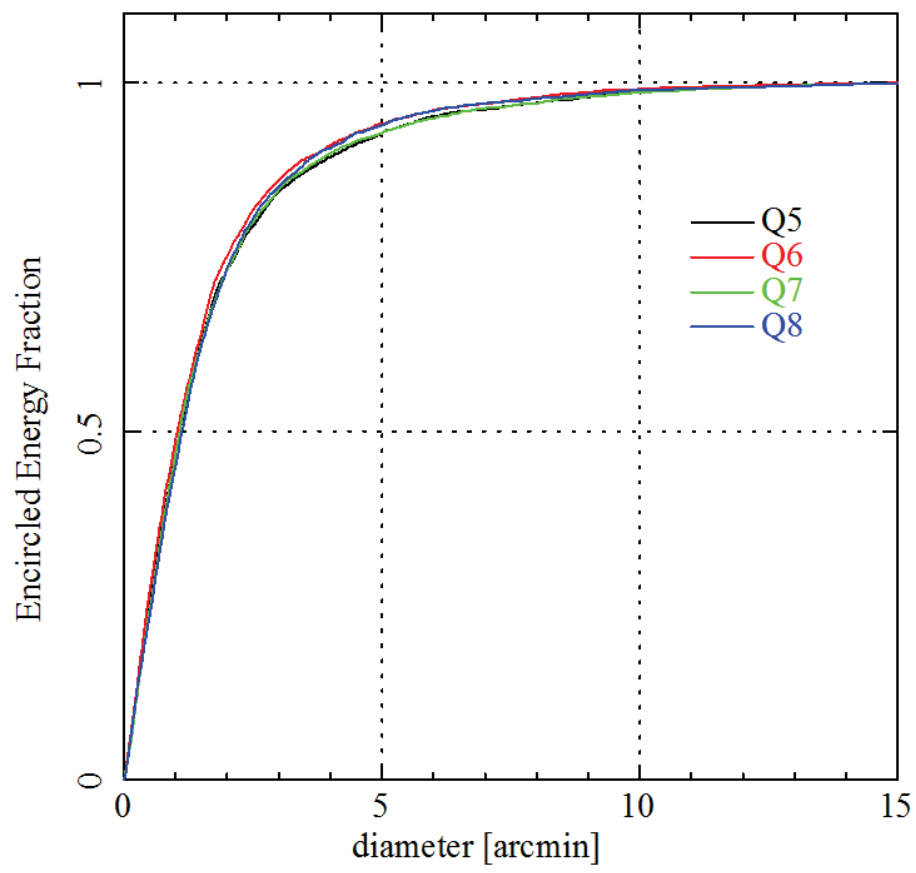

Fig. $10 \mathrm{X}$-ray HPD of the four quadrants of FM2. The angular resolution is very similar among the four

\section{DISCUSSIONS}


Quality of the flight mirrors is similar with each other. It is largely due to the uniform quality of the housing and alignment structure, and the consistent quality of the flight reflectors. The assembly procedures are well established and being followed well in assembly of both mirrors.

Vibration test: the FM1 went through the vibration test at the acceptance level. The image quality of the FM1 at quadrant and mirror level was verified after the vibration. The change was insignificant and there were no observable damages of the reflectors and the supporting structure. FM2 will be vibrated in early September before delivery to Japan for further testing and integration to the spacecraft.

As we demonstrated in the study, figure/roundness errors of the reflectors, which in turn contribute to the image $\sim 1$ arc-minute RMS, is the dominant one. The assembly errors, either in the hardware specs or procedures under environmental influences, are of a similar magnitude. Apparently both of these dominant errors terms have to be dealt with before the angular resolution can be improved. From the PSF in Fig. 8, we can observe that the power in the 40-100 arc-second range has to be reduced in order to obtain a better angular resolution.

Beyond the quadrant level, assembly focuses on the confocality of the quadrants and the parallelism of the four quadrants optical axes, which have little impact on the image quality at the whole mirror level.

\section{CONCLUSIONS}

- High throughput X-ray mirrors with image quality at 1 arc-minute provide an excellent tool to study spectroscopy of the celestial X-ray sources

- Thin-foil X-ray mirror technology made a 2-fold improvement on angular resolution over the Suzaku mirrors

- The integrated mirror FM1 has been delivered to JAXA/ISAS, and FM2 is nearly being completed at Goddard before the September delivery. The image quality of the integrated mirror is largely determined by the quality of their individual quadrant. Errors that being introduced in the mirror integration was proven to be minimal

- As the reflector design/fabrication and assembly hardware/scheme keep on improving, the angular resolution of this affordable imagers may achieve at HPD 10-20 arc-seconds level in the near future

\section{ACKONWLEDGEMENTS}

Authors would like to thank the ASTRO-H production crews, including Cliff Brown, Dominic Contino, John Kearney, Larry Lozzipone, Ai Numata, Paul Pless, Mark Schofield, Emory Toomey III, for their great contribution on fabricating/logging the reflectors, Lawrence Olsen who coordinates the lab operation, T. Hayashi, Joseph Sytwu, Devin Hahn (currently at Applied Physics Laboratory) and Nagoya group led by Dr. Y. Tawara on integrating the FM, and NASA/MSFC and Harvard SAO X-ray optics groups on technical exchanges.

\section{REFERENCES}

[1] Peter J. Serlemitsos, Yang Soong, Takashi Okajima, and Devin J. Hahne, "Foil X-ray Mirrors for Astronomical Observations: Still an Evolving Technology", Proc. SPIE, 7732, 77320A, 2010, and references therein

[2] Peter J. Serlemitsos, Yang Soong, "Foil X-ray Mirrors”, Astrophysics and Space Science, 239, 177, 1996

[3] Gubarev, Mikhail V., MSFC group for SXT-I, private communication, 2010

[4] Takashi Okajima, Peter J. Serlemitsos, Yang Soong, "Performance of the ASTRO-H Soft X-ray Telescope (SXT-1), AAS, HEAD \#13, \#123.24, 2013 